\title{
Stiff Heart Syndrome
}

Satya S. Bhupathi, MD, MPH; Sreelatha Chalasani, MD, MPH; and Roxann Rokey, MD, FACC, FASE

\begin{abstract}
Isolated cardiac amyloidosis, or "Stiff Heart Syndrome," is a rare manifestation of amyloidosis. Some degree of cardiac amyloid deposition is common in elderly patients, as reported in prior post-mortem studies; however, isolated cardiac involvement with predominantly cardiac symptoms and no evidence of systemic disease is a rare presentation. Establishing the correct diagnosis, even with the use of extensive testing including amyloid typing, understanding the clinical significance, and management can be challenging in such cases.
\end{abstract}

Keywords: Cardiac amyloidosis; Stiff heart syndrome; Diagnosis; Management

Corresponding Author:

Sreelatha Chalasani, MD, MPH

Department of Internal Medicine

Marshfield Clinic

1000 North Oak Avenue

Marshfield, WI 54449

Tel.: 715-387-5537

Fax: 715-389-5757

E-mail: chalasani.sreelatha@marshfieldclinic.org

Received: November 10, 2010

Revised: June 25, 2010

Accepted: August 4, 2010

doi: $|0.3| 2 \mid / \mathrm{cmr} .2010 .899$
$\mathrm{H}$ incidence and prevalence. Ischemic cardiomyopathy resulting from coronary artery disease is the most common cause of heart failure in the United States ${ }^{1}$. Non-ischemic cardiomyopathy is characterized by the absence of coronary artery disease and includes dilated, hypertrophic, and restrictive causes of cardiomyopathy. Out of these three types, restrictive cardiomyopathy is rare in the United States and most other industrialized nations. Restrictive cardiomyopathy is characterized by stiffening of the ventricular walls and loss of myocardial flexibility due to infiltration by abnormal tissue, resulting in inadequate ventricular filling with blood and eventually the loss in its ability to pump properly.

Restrictive cardiomyopathy is involved in approximately $5 \%$ of all primary myocardial diseases and can be due to either idiopathic or secondary causes. Amyloidosis, hemochromatosis, and sarcoidosis are among the most frequently encountered causes of secondary restrictive cardiomyopathy. Apart from these, secondary restrictive cardiomyopathy is also caused by primary systemic sclerosis, carcinoid heart disease, glycogen storage disease of the heart, radiationinduced heart disease, metastatic malignancy, anthracycline toxicity, endomyocardial fibrosis, and Loeffler eosinophilic endomyocardial disease. Restrictive cardiomyopathy shares similarities in clinical and hemodynamic profiles with constrictive pericarditis. Due to the difference in management, accurate diagnosis and differentiation of these two conditions is necessary. We present two cases of isolated cardiac amyloidosis.

\section{Case Presentations}

\section{Case 1}

A man, aged 74 years, was referred to the cardiology clinic following a witnessed episode of syncope. The patient described his syncopal episodes, which have been occurring over several years, as sudden onset with occasional symptoms of 


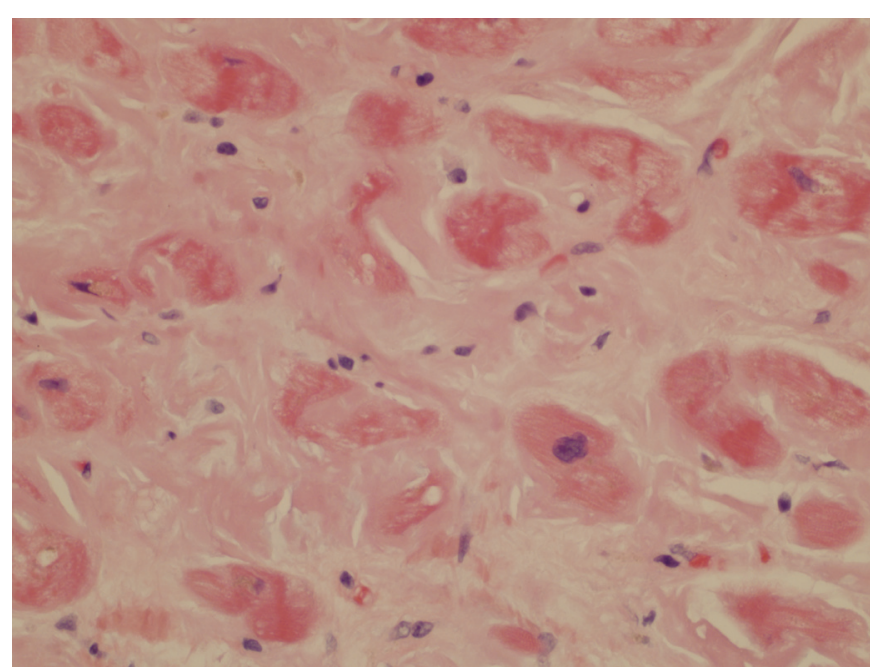

Figure 1. Endomyocardial biopsy showing extensive amyloid deposition as pale eosinophilic material surrounding myocardial cells (H\&E, x40).

light-headedness. He denied symptoms of chest pain and/or discomfort, palpitations, orthopnea, paroxysmal nocturnal dyspnea, or recent change in exercise tolerance. Significant past medical history includes only hyperlipidemia. He does not smoke, uses alcohol rarely, and denies significant exposure to chemicals. Physical examination was normal, except for the cardiac examination which showed cardiomegaly. Laboratory evaluations were within normal limits. Transthoracic echocardiogram revealed mild to moderate concentric left ventricular (LV) hypertrophy with no pericardial effusion, intracardiac masses, shunts, clots or vegetation. Dimensions of the cardiac chambers showed normal left atrium with enlarged right atrium. The inter-ventricular septum was slightly thickened, while the left ventricular end diastolic dimension and right ventricular end systolic dimensions were normal. A tilt-table test was performed, including infusion of isoproterenol, and was non-diagnostic of orthostatic hypotension.

As the patient continued to experience light-headedness, a work-up for restrictive cardiomyopathy was initiated. Serum protein immuno-electrophoresis and serum free light chain analysis were normal. Fat pad biopsy showed no histopathologic abnormality and the Congo red stain for amyloid was negative. Subsequently, the patient underwent a coronary angiogram, left ventriculography, and right ventricle biopsy along with right and left heart catheterization. Apart from $30 \%$ stenotic lesions in the proximal right coronary, and proximal and mid left anterior descending artery, the remaining coronary arteries were disease free. Biplane left ventriculography showed mild global LV hypokinesia. The endomyocardial biopsy showed diffusely infiltrated myocardium with waxy, pale, eosinophilic material showing green birefringence under standard polarized light and red under fluorescent light with a Texas red filter, characteristic of amyloid (figure 1). Positive Congo red and sulfated Alcian blue stains confirmed the presence of amyloid deposition. Immunohistochemical studies were performed on paraffin sections using antibodies directed against serum amyloid $\mathrm{P}$ component, transthyretin, kappa and lambda immunoglobulin free light chains, and serum amyloid A. The amyloid deposits showed strong staining for transthyretin, with negative staining for serum amyloid P, kappa and lambda light chains and for serum amyloid A. These results were consistent with transthyretin-type amyloid deposition, which could represent either senile or familial amyloidosis. Bone marrow aspiration, performed to rule out plasma cell dyscrasias, showed normocellular marrow with no evidence of amyloid deposition.

The patient was diagnosed with "Isolated Cardiac Amyloidosis" or "Stiff Heart Syndrome," with senile amyloid deposition. He was told there was no evidence of light chain disease, and that this amyloidosis does not respond to chemotherapeutic interventions, but does have a relatively favorable prognosis compared to amyloid light chain (AL) cardiac amyloid. Median survival in this group of patients without any chemotherapy is about seven years. Due to the relatively favorable prognosis in this patient, no chemotherapy was initiated, and he continues follow-up care in the cardiology clinic.

\section{Case 2}

A man, aged 74 years, was referred to the cardiology clinic for a newly diagnosed congestive heart failure (CHF). The patient presented with a 3-month history of progressively worsening exertional dyspnea with marked physical activity limitation and bilateral symmetric lower extremity edema. The patient denied symptoms of chest pain, palpitations, orthopnea, paroxysmal nocturnal dyspnea, presyncopal symptoms or syncope. He had no history of significant occupational chemical exposure and did not smoke or use alcohol. He had no history of hypertension, diabetes mellitus, hyperlipidemia, or thyroid dysfunction.

Pulmonary examination revealed decreased breath sounds at the bilateral bases, with the left more significant than the right. Cardiac examination showed a non-displaced point of maximal impact. In the sitting position on forced expiration, a mild aortic insufficiency murmur was auscultated. The remainder of his system review was normal.

Echocardiography (2D-echocardiography) showed depressed LV systolic function with regional wall motion abnormalities involving the septal and inferior walls. Chest radiograph showed bilateral pleural effusions with non-acute pulmonary parenchymal disease. He was started on an angiotensin converting enzyme inhibitor (lisinopril) and diuretic (torsemide) prior to this referral.

The patient underwent elective coronary angiography and left ventriculography with right and left heart catheterization. Biplane left ventriculography showed diffuse LV hypokinesia. 




Figure 2. Endomyocardial biopsy showing extensive amyloid deposition as pale eosinophilic material surrounding distorted myocardial cells (H\&E, x40).

Overall, the findings were non-diagnostic for either pure restrictive or constrictive physiology, although mild restrictive physiology could not be completely ruled out. The patient was diagnosed with idiopathic cardiomyopathy and started on a beta blocker (metoprolol succinate) and digoxin along with his prior medications. A 2-month follow-up echocardiogram showed normal LV function, normal regional wall motion, normal LV ejection fraction (LVEF), and moderate LV hypertrophy. He returned to his primary care physician for heart failure management.

Three years after being diagnosed with idiopathic cardiomyopathy, the patient was re-referred to cardiology with a 2-month history of acutely decompensated heart failure symptoms and epigastric discomfort. Resting electrocardiogram showed atrial fibrillation (AF) with ventricular rate of $93 \mathrm{bpm}$ and no associated sign of ischemia, injury, or infarction. Repeat laboratory evaluations were within normal limits. The 2D-echocardiography was repeated, showing mean right heart pressures, left heart pressures, cardiac output, and cardiac index were comparably similar to earlier findings. These findings suggested that the patient had a repeat myopathic process of unclear etiology, now in the presence of AF.

After one month of therapeutic anticoagulation with warfarin, the patient underwent trans-esophageal echocardiography. The transesophageal echocardiography revealed LVEF of $35 \%$ and no thrombus. The patient's rhythm was converted to normal sinus rhythm by electric-cardio-version using a single shock of 300-joules of synchronized direct current. Also, an abdominal fat pad biopsy was performed, which showed normal adipose tissue and negative Congo red staining with no histological evidence for other infiltrative diseases.
This patient continued to have decompensated CHF with New York Heart Association (NYHA) Class III-IV symptoms. Repeat right heart catheterization with endomyocardial biopsy was performed. The histopathological findings of the biopsy were highly consistent for amyloidosis (figure 2 ) with positive Congo red staining and apple green birefringence (figure 3). The patient was diagnosed with "Isolated Cardiac Amyloidosis" or "Stiff Heart Syndrome."

Considering the patient's age and co-morbidities, he was not found to be an appropriate candidate for heart-lung transplant. He underwent chemotherapy with MDex (melphalan and dexamethasone). Chemotherapy with MDex is standard therapy for primary AL amyloidosis patients who have not undergone or are not appropriate candidates for stem cell transplant. ${ }^{2}$ Unfortunately, after the first cycle of chemotherapy, he developed melena requiring blood transfusion. After considering risk versus benefit with poor tolerance to chemotherapy, it was agreed to discontinue further chemotherapy and continue medical monitoring.

Five years after the initial onset of heart failure and twelve months after the biopsy-proven diagnosis of isolated cardiac amyloidosis, or stiff heart syndrome complicated by AF, he continues to follow-up in the cardiology clinic. His heart failure is compensated with NYHA Heart Failure Class II, currently on furosemide, lisinopril and metolazone. For AF, rate control is achieved with carvedilol along with continuation of anticoagulation.

\section{Discussion}

Amyloidosis is a relatively uncommon, mysterious, and under-diagnosed disease associated with extracellular deposition of amorphous, homogenous, insoluble, fibrillar proteinaceous material in various organs of the body. Several types of proteins including light chain immunoglobulins, transthyretin, acute-phase reactants, and apoprotein A have been reported to cause amyloidosis. ${ }^{3}$ All amyloid deposits share a characteristic microscopic beta pleated structure with positive Congo red staining and apple green bifringence under polarizing light. ${ }^{3}$ Liver, spleen, and kidney are the most commonly associated organs, however amyloid depositions have been identified in virtually every organ of the body. Symptoms and clinical presentation are characterized by the type of deposition and organ of significant involvement.

Cardiac amyloidosis or "stiff heart syndrome" is characterized by extracellular amyloid infiltration of the myocardial tissue. Though deposition of amyloid in the hearts of elderly asymptomatic populations is not uncommon, symptomatic isolated cardiac amyloidosis is infrequent. ${ }^{4}$ Frequently, cardiac amyloidosis coexists with evident dysfunction of other major organs and is identified with multi-organ involvement. Symptoms associated with cardiac amyloidosis simulate cardiomyopathy, coronary heart disease, valvular heart disease, coronary artery disease, or arrhythmia. ${ }^{5-7}$ It is estimated that $10 \%$ of cases of non-ischemic cardiomyopathy 




Figure 3. Orange-pink staining amyloid which exhibited yellow-green birefringence under polarized light (not shown) (Congo red, x40).

are secondary to cardiac amyloidosis. ${ }^{8}$ Frequently reported symptoms of cardiac amyloidosis are predominantly associated with right heart failure, including atypical chest pain, dyspnea on exertion, fatigue, peripheral edema, and palpitations. Purpura is another frequently reported symptom of cardiac amyloidosis, but typically only in primary $\mathrm{AL}$ amyloidosis patients. ${ }^{6}$ By the time a patient becomes symptomatic, it is estimated that $25 \%$ of the myocardial mass is infiltrated with amyloid deposition. ${ }^{10}$ However, due to the high prevalence of coronary heart disease, it is imperative to rule this out prior to initiating workup for cardiac amyloidosis. Isolated cardiac amyloidosis, without clear cut evidence of other organ involvement, is possible. ${ }^{9}$ Iqbal et $\mathrm{al}^{11}$ reported a unique case with amyloid deposition on the aortic valve, with no evidence of systemic disease. In an autopsy study of 100 patients, isolated atrial amyloid deposition was identified in more than $75 \%$ of patients over age $50 .{ }^{12}$ These depositions were of a higher grade in females when compared to males and associated with a risk of chronic $\mathrm{AF} .{ }^{13}$ Chronic $\mathrm{AF}$ is noted in $10 \%$ to $20 \%$ of cases with cardiac amyloidosis. ${ }^{13}$

Over the years, several types of protein depositions have been identified and linked to subtypes of amyloidosis. ${ }^{7}$ Almost all forms of amyloidosis, including primary $\mathrm{AL}$, hereditary, and "senile" systemic can cause cardiomyopathy and/or heart failure. The most common type associated with cardiac involvement (up to $85 \%$ ) is primary amyloidosis, associated with the deposition of AL amyloid protein. This protein is made of either lambda- $(\operatorname{Ig} \lambda)$ or kappa- $(\operatorname{Ig} \kappa)$ light chain immunoglobulins. ${ }^{13}$ Primary amyloidosis is the most common form of amyloidosis, involves more organs, progresses quickly, and is commonly seen in patients with multiple myeloma. ${ }^{14}$ Familial amyloidosis, such as ApoA1 type, and "senile" systemic amyloidosis, such as TTR type, are the second most frequent types and are caused by the deposition of transthyretin or ATTR amyloid protein, and are associated with $10 \%$ of cardiac amyloidosis cases. ${ }^{15,16}$ Cardiac amyloidosis secondary to chronic inflammation due to diseases like rheumatoid arthritis, tuberculosis, and Crohn's disease is rare. This form of amyloidosis is associated with the deposition of an acute phase reactant protein called serum amyloid A protein. ${ }^{7,17}$ Secondary amyloidosis almost never affects the heart in a clinically significant manner., ${ }^{7,18}$ Isolated atrial amyloidosis results in amyloid depositions which are immunoreactive for atrial natriuretic peptide. In one study, out of 245 atrial appendages isolated from patients undergoing open heart surgery, Röcken et $\mathrm{al}^{19}$ reported $16.3 \%$ (40 of 245) of cases as having amyloid deposition. Of these 245 patients, 38 $(15.5 \%)$ had $\mathrm{AF}$ with a statistically significant correlation independent of age and gender for the association of amyloid deposition and atrial fibrillation. ${ }^{19}$ Cardiac involvement in the other subtypes of amyloidosis, including dialysis-associated amyloidosis, familial Mediterranean fever, and familial corneal amyloidosis, is rare and generally a late development in the disease process. ${ }^{20-23}$

Increased risk of AF has been reported with AL amyloidosis, senile cardiovascular amyloidosis and isolated atrial amyloidosis. Interstitial, perivascular, or endocardial deposits of amyloid proteins causes atrial fibrosis, which is known to increase the risk of AF. Atrial natriuretic peptide deposited in isolated atrial amyloidosis has been shown to have arrythmogenic properties increasing risk of atrial fibrillation, especially as it leads to the prolongation of the P-wave duration. ${ }^{19}$

Symptoms and signs of systemic, as well as cardiac, amyloidosis are mostly non-specific. This is further confounded by the high prevalence of other cardiovascular diseases such as coronary heart disease, arrhythmias, and valvular heart disease. High clinical suspicion for restrictive etiology of unexplained cardiomyopathy is essential and requires a multidisciplinary approach for accurate diagnosis and treatment. A thorough clinical history, physical examination, biochemical analysis, imaging studies, genetic analysis, and histopathological evaluation are essential. Due to the similarity in clinical presentation to constrictive pericarditis and the differences in management, accurate time-sensitive diagnosis is important. Physical findings like distant heart sounds, elevated jugular pressures, blunted $\mathrm{y}$-descent, pulses paradoxus, and other right heart failure signs must be evaluated with high clinical suspicion. Electrocardiogram with low voltage in limb leads, Q waves, arrhythmias, and signs of increased left ventricular mass have moderate sensitivity of $63 \%$ to $80 \%{ }^{24}$ Echocardiographic evidence of dilated atria, interatrial septum $>7 \mathrm{~mm}$, thickened interventricular septum, sparkled appearance of myocardium, and presence of pericardial effusion have varying sensitivity for the diagnosis of cardiac amyloidosis. ${ }^{17,25}$ Pulse and colorcoded tissue Doppler imaging has been used to measure myocardial function and myocardial velocity profile. Tissue Doppler imaging can also calculate the myocardial velocity gradient across the interventricular septum and posterior left 
ventricular walls. Characteristic serrated appearance in the M-mode has been shown to have high diagnostic value in patients with cardiac amyloidosis. ${ }^{26}$

Coronary angiography is primarily intended to rule out coronary artery disease, but should not be used if the patient is experiencing cardiorenal problems. ${ }^{27}$ Cardiac catheterization with full hemodynamic evaluation is essential, but is generally done at the time of coronary artery evaluation or endomyocardial biopsy. Impaired left ventricular relaxation with elevated left ventricular end-diastolic pressure is a common association. ${ }^{28}$ Hemodynamic tracings help in making the distinction from constrictive pericarditis, which is often associated with equalization of the right and left ventricular pressures.

Basic blood counts, inflammatory markers like erythrocyte sedimentation rate, serum cardiac biomarkers (BNP and troponin), chest radiography, urine and serum protein electrophoresis, urine and serum immunofixation electrophoresis, thyroid function testing, serum amyloid A protein, transthyretin level, and serum free light chain levels all have diagnostic value..$^{7,9,13,29,30}$ Endomyocardial biopsy has $75 \%$ sensitivity in accurately diagnosing cardiac amyloidosis; however, this can be avoided in the presence of other diagnostic evidence, except in the case of isolated cardiac amyloidosis. ${ }^{16,31}$ According to the American College of Cardiology, American Heart Association, and the European Society of Cardiology 2007 heart failure guidelines, endomyocardial biopsy is a class IIa recommendation for assessing heart failure patients. ${ }^{27}$ Endomyocardial biopsy should not be performed in the routine evaluation of patients with heart failure, but can be useful in patients presenting with heart failure when a specific diagnosis, such as heart failure associated with unexplained restrictive cardiomyopathy, is suspected that would influence therapy. ${ }^{27}$

Fat pad aspiration, gingival, rectal, bone marrow, sural nerve, skeletal muscle, and gastrointestinal biopsies can be used for the diagnosis of systemic amyloidosis. ${ }^{32-36}$ Fat pad biopsy is a useful and important screening procedure for systemic amyloidosis. In a retrospective analysis published by Dhingra et $\mathrm{al},{ }^{37}$ abdominal fat pad fine needle aspiration cytology showed a sensitivity of $78 \%$, specificity of $93 \%$, positive predictive value of $84 \%$, and negative predictive value of $90 \%$. Sensitivity is further increased by evaluating multiple smears. Gameren et $\mathrm{al}^{38}$ reported that routine assessment of single fat pad smear resulted in sensitivity of $80 \%$. However, by using an approach to thoroughly examine three smears by two observers, the sensitivity increased to $93 \%$.

For typing amyloidosis, immunofixation studies have better diagnostic value in differentiating from plasma cell dyscrasias than immunohistochemistry. ${ }^{39}$ Further improvement in sensitivity is achieved by testing with the quantitative serum free light chains assay which has a higher sensitivity compared to immunofixation. ${ }^{40}$ The kappa/lambda free light chain ratio obtained in this test helps in establishing the diagnosis, especially in cases with co-existing renal impairment. Since both immunofixation and the serum free light chains assay detect a monoclonal protein, but cannot prove that it is responsible for the formation of the amyloid deposits, they should both be used to maximize diagnostic sensitivity. ${ }^{14}$ The availability of genetic testing has also improved the ability to type amyloidosis.

A highly specific and sensitive test for typing amyloidosis was recently developed for clinical biopsy specimens, combining laser microdissection sampling with tandem mass spectroscopy-based proteomics analysis. ${ }^{41}$ These newer techniques enhance the ability to accurately type amyloidosis, which is required in the determination of the most effective treatment regimens.

Morphologically, amyloid hearts are heavier, enlarged, waxy, firm, and rubbery with thickened walls. Light microscopy shows amorphorous deposits by hematoxylin and eosin staining. The two characteristic features of amyloid deposition are orange- or red-colored tinge with Congo red and apple green bifringence with polarized light. To type the amyloid deposits after positive Congo red staining, special immunohistochemical staining studies are recommended. Commercially available anti-amyloid fibril protein antibodies against $\kappa$ light chains, $\lambda$ light chains, AA amyloid, $\beta_{2}$ macroglobulin, atrial natriuretic peptide, and transthyretin are used. ${ }^{42,43}$

It is postulated that deposition of human amyloidogenic light chain in the myocytes leads to increased oxidative stress, resulting in reduced contractility, reduced relaxation, and alteration in intracellular calcium handling. ${ }^{44}$ Liao et $\mathrm{al}^{45}$ isolated light chains from patients with primary amyloidosis (AL), with and without cardiac involvement, to study their effect on isolated mouse hearts. The study results showed that infusion of light chains from patients without cardiac involvement resulted in the development of diastolic dysfunction similar to patients with cardiac involved $\mathrm{AL}$ amyloidosis. These results suggest that amyloid light chain proteins deposition can cause rapid progression of cardiomyopathy, independent of extracellular amyloid fibrillar deposition. Even in primary systemic amyloidosis, CHF remains the primary cause of death. ${ }^{46}$

Once the diagnosis of cardiac amyloidosis is established, management of these patients involves symptom control and treatment of underlying disease process. Congestive heart failure symptoms are treated with diuretics. Calcium channel blockers are contraindicated as they are associated with increased toxicity and significant ionotropic effect. ${ }^{47}$ Angiotensin-converting enzyme inhibitors and angiotensin receptor blockers have been associated with increased risk of hypotension and should be used with extreme caution. Beta blockers have not been shown to improve survival and have side effects similar to angiotensin converting enzyme inhibitors and angiotensin receptor blockers. Midodrine has 
been used successfully to treat orthostatic hypotension in patients with cardiac amyloidosis. ${ }^{48}$ In cases with concomitant AF, digoxin can be used with caution. ${ }^{7}$ However, the propensity of digoxin to bind with amyloid protein fibrils increases its availability and causes digoxin toxicity, even at therapeutic digoxin levels. ${ }^{49}$ Pacemakers have been used in patients with symptomatic bradycardia. The atrial and ventricular pacing thresholds have been shown to be higher in these patients. In patients with AF, aspirin and anticoagulation are strongly recommended due to the increased propensity to form atrial thrombi even after converting to sinus rhythm. The poor atrial function due to infiltration with amyloid material and small mitral A wave noted on transesophageal echocardiography $\left(\leq 20 \mathrm{~cm} / \mathrm{sec}\right.$ ) are strong predictors for this recommendation. ${ }^{50,51}$ The safety and efficacy of antiarrythemic agents and implantable defibrillators in preventing malignant arrhythmias has not been studied in cardiac amyloidosis patients.

Chemotherapy regimens to treat amyloidosis have been evolving over the last few decades; however, it has better efficacy in treating AL type amyloidosis with evidence of an underlying amyloid-forming plasma cell clone. ${ }^{39}$ In the period known as the "colchicine era" (1980-1987), the mainstay of treatment was monotherapy with colchicine. ${ }^{52}$ Subsequently, the MPC trial results led to the use of melphalan, prednisone and colchicine as a combined regimen. In recent years, melphalan along with stem cell transplant has become the mainstay of treatment in patients who can tolerate it. ${ }^{53}$ The same regimens have been used in cardiac amyloidosis. Patients with severe symptoms showing no improvement with the above therapy have received palliative heart transplants or cardiac allograft followed by stem cell transplant. ${ }^{2}$ This is more common in cardiac amyloidosis with primary AL amylodosis and less so with secondary or familial subtypes of the disease. ${ }^{54}$ Although, in the U.S.A. and Europe, patients with hereditary amyloidosis caused by variant transthyretin who are young and have minimal symptoms, are usually considered candidates for organ transplant. ${ }^{2}$ Ongoing studies are evaluating immunotherapeutic agents, such as monoclonal antibodies, to treat this disease. It is postulated that an amyloid-reactive monoclonal antibody should stimulate fibril disassembly. ${ }^{55}$ Etanercerpt has been shown to provide limited benefits in cardiac amyloidosis. ${ }^{55}$ Use of similar agents with mixed results has been reported, and these agents have limited efficacy in cases with cardiac amyloidosis. Other new therapies, especially for secondary amyloidosis, are currently being evaluated in clinical trials.

Prognosis in patients with cardiac amyloidosis depends on the subtype. Amyloid light-chain amyloidosis has a poor prognosis with median survival of 13 months without treatment. After the onset of $\mathrm{CHF}$ in $\mathrm{AL}$ amyloidosis, median survival is only six months. ${ }^{56}$ Poor prognostic factors include increased left ventricular wall thickness, syncope, and elevated troponin I and $\mathrm{T}$. In patients with cardiac amyloidosis, median survival improves to 18 months with treatment. Wide variations of average survival after heart transplantation have been reported, ranging from 32 to 118 months in various case reports and case series. ${ }^{57}$ Prognosis in secondary amyloidosis with cardiac involvement is dependent on the treatment of the primary disease and has an average 5-year survival rate of $31 \%{ }^{58}$ Prognosis with isolated atrial amyloidosis is not documented.

\section{Conclusion}

Amyloidosis is a mysterious and complex disease that requires high clinical suspicion for diagnosis. Earlier diagnosis would mitigate end organ dysfunction and improve prognosis. Cardiac amyloidosis should be considered in the differential diagnosis of all patients with characteristic echocardiographic findings and correlating symptoms of right heart, especially in the absence of coronary artery disease. In our two cases, abdominal fat pad biopsy was negative for systemic amyloidosis. However, endomyocardial biopsy was pursued due to high clinical suspicion for infiltrative cardiomyopathy, resulting in the final diagnosis of isolated cardiac amyloidosis. Endomyocardial biopsy with appropriate immunohistochemical staining is a useful tool for diagnosis of this condition. Recent advances in chemotherapy, stem cell transplantation, and immunotherapy are not curative but help improve survival.

\section{Acknowledgments}

The authors thank Dr. Gene R. Shaw of the Marshfield Clinic Pathology Department for supplying the pathology photos and the Marshfield Clinic Research Foundation's Office of Scientific Writing and Publication for writing and editorial assistance in the preparation of this article.

\section{References}

1. Massie B, Shah N. Evolving trends in the epidemiologic factors of heart failure: rationale for preventive strategies and comprehensive disease management. Am Heart J 1997; 133:703-712.

2. Comenzo RL. Managing systemic light-chain amyloidosis. J Natl Compr Canc Netw 2007;5:179-187.

3. Merlini G, Westermark P. The systemic amyloidoses: clearer understanding of the molecular mechanisms offers hope for more effective therapies. J Intern Med 2004;255:159-178.

4. Sugiura M. Characteristic features of the heart disease in the elderly. [Article in Japanese] Nippon Ronen Igakkai Zasshi 1994;31:182-186.

5. Kholova I, Niessen HW. Amyloid in the cardiovascular system: a review. J Clin Pathol 2005;58:125-133.

6. Kyle RA, Bayrd ED. Amyloidosis: a review of 236 cases. Medicine (Baltimore) 1975;54:271-299.

7. Falk RH, Comenzo RL, Skinner M. The systemic amyloidosis. N Engl J Med 1997;337:898-909.

8. Kingman A, Pereira NL. Cardiac amyloidosis. J S C Med Assoc 2001;97: 201-206.

9. Lindholm PF, Wick MR Isolated cardiac amyloidosis associated with sudden death. Arch Pathol Lab Med 1986;110:243-245.

10. Roberts WC, Waller BF. Cardiac amyloidosis causing cardiac dysfunction: analysis of 54 necropsy patients. Am J Cardiol 1983;52:137-146.

11. Iqbal S, Reehana S, Lawrence D. Unique type of isolated cardiac valvular amyloidosis. J Cardiothorac Surg 2006;1:38.

12. Steiner I, Hajkova P. Patterns of isolated atrial amyloid: a study of 100 hearts on autopsy. Cardiovasc Pathol 2006; $15: 287-290$. 
13. Dubrey SW, Cha K, Anderson J, Chamarthi B, Reisinger J, Skinner, Falk RH. The clinical features of immunoglobulin light-chain (AL) amyloidosis with heart involvement. QJM 1998;91:141-157.

14. Comenzo RL. How I treat amyloidosis. Blood 2009; 114:3147-3157.

15. Hattori T, Takei Y, Koyama J, Nakazato M, Ikeda S. Clinical and pathological studies of cardiac amyloidosis in transthyretin type familial amyloid polyneuropathy. Amyloid 2003;10:229-239.

16. Kothari SS, Ramakrishnan S, Bahl VK. Cardiac amyloidosis-an update. Indian Heart J 2004;56:197-203.

17. Dubrey SW, Cha K, Simms RW, Skinner M, Falk RH. Electrocardiography and Doppler echocardiography in secondary (AA) amyloidosis. Am J Cardiol 1996;77:313-315.

18. Falk R. Diagnosis and management of the cardiac amyloidoses. Circulation 2005;112:2047-2060.

19. Röcken C, Peters B, Juenemann G, Saeger W, Klein HU, Huth C, Roessner A, Goette A. Atrial amyloidosis: an arrhythmogenic substrate for persistent atrial fibrillation. Circulation 2002;106:2091-2097.

20. Takayama F, Miyazaki S, Morita T, Hirasawa Y, Niwa T. Dialysis-related amyloidosis of the heart in long-term hemodialysis patients. Kidney Int Suppl 2001;78:S172-S176.

21. Varga J, Wohlgethan JR. The clinical and biochemical spectrum of hereditary amyloidosis. Semin Arthritis Rheum 1988; 18:14-28.

22. Sohar E, Pras M, Heller J, Heller H. Genetics of familial Mediterranean fever. Arch Intern Med 1961;107:529-538.

23. Gorevic PD, Rodrigues MM. Ocular amyloidosis. Am J Opthhalmol 1994;117:529-532.

24. Rahman JE, Helou EF, Gelzer-Bell R, Thompson RE, Kuo C, Rodriguez ER, Hare JM, Baughman KL, Kasper EK. Noninvasive diagnosis of biopsy-proven cardiac amyloidosis. J Am Coll Cardiol 2004;43:410-415.

25. Cueto-Garcia L, Reeder GS, Kyle RA, Wood DL, Seward JB, Naessens J, Offord KP, Greipp PR, Edwards WD, Tajik AJ. Echocardiographic findings in systemic amyloidosis: spectrum of cardiac involvement and relation to survival. J Am Coll Cardiol 1985;6:737-743.

26. Oki T, Tanaka H, Yamada H, Tabata T, Oishi Y, Ishimoto T, Nagase N, Shinohara H, Sakabe K, Fukuda N. Diagnosis of cardiac amyloidosis based on the myocardial velocity profile in the hypertrophied left ventricular wall. Am J Cardiol 2004;93:864-869.

27. Hunt SA, Abraham WT, Chin MH, Feldman AM, Francis GS, Ganiats TG, Jessup M, Konstam MA, Mancini DM, Michl K, Oates JA, Rahko PS, Silver MA, Stevenson LW, Yancy CW, Antman EM, Smith SC Jr, Adams CD, Anderson JL, Faxon DP, Fuster V, Halperin JL, Hiratzka LF, Jacobs AK, Nishimura R, Ornato JP, Page RL, Riegel B; American College of Cardiology; American Heart Association Task Force on Practice Guidelines; American College of Chest Physicians; International Society for Heart and Lung Transplantation; Heart Rhythm Society. ACC/AHA 2005 guideline update for the diagnosis and management of chronic heart failure in the adult: a report of the American College of Cardiology/American Heart Association Task Force on Practice Guidelines (Writing Committee to Update the 2001 Guidelines for the Evaluation and Management of Heart Failure). J Am Coll Cardiol 2005; 46(6): 1116-1143.

28. Swanton RH, Brooksby IA, Davies MJ, Coltart DJ, Jenkins BS, Webb-Peploe MM. Systolic and diastolic ventricular function in cardiac amyloidosis. Studies in six cases diagnosed with endomyocardial biopsy. Am J Cardiol 1977;39:658-664.

29. Pascali E. Diagnosis and treatment of Primary Amyloidosis. Crit Rev Oncol Hematol 1995;19:149-181.

30. O'Hara CJ, Falk RH. The diagnosis and typing of cardiac amyloidosis. Amyloid 2003;10:120-129.
31. Ardehali H, Qasim A, Cappola T, Howard D, Hruban R, Hare JM, Baughman KL, Kasper EK. Endomyocardial biopsy plays a role in diagnosing patients with unexplained cardiomyopathy. Am Heart J 2004;147:919-923.

32. Hunt SA, Hutter Jr AM. Heart failure: Best inotrope, best vasodilator, indications for biopsy. Am Coll Cardiol Cardiosource 2009; 01/27/2009. Available at: www.medscape.com/ viewarticle/586488. Accessed October 20, 2009.

33. Gertz MA. Diagnosing primary amyloidosis. Mayo Clin Proc 2002;77:1278-1279.

34. Kuroda T, Tanabe N, Sakatsume M, Nozawa S, Mitsuka T, Ishikawa H, Tohyama CT, Nakazono K, Murasawa A, Nakano M, Gejyo F. Comparison of gastroduodenal, renal and abdominal fat biopsies for diagnosing amyloidosis in rheumatoid arthritis. Clin Rheumatol 2002;21:123-128.

35. Gafni J, Sohar E. Rectal biopsy for the diagnosis of amyloidosis.Am J Med Sci 1960;240:332-336.

36. Rajani B, Rajani V, Prayson RA. Peripheral nerve amyloidosis in sural nerve biopsies: a clinicopathologic analysis of 13 cases. Arch Pathol Lab Med 2000;124:114-118.

37. Dhingra S, Krishnani N, Kumari N, Pandey R . Evaluation of abdominal fat pad aspiration cytology and grading for detection in systemic amyloidosis. Acta Cytol 2007; 51:860-864.

38. van Gameren II, Hazenberg BP, Bijzet J, van Rijswijk MH. Diagnostic accuracy of subcutaneous abdominal fat tissue aspiration for detecting systemic amyloidosis and its utility in clinical practice. Arthritis Rheum 2006;54:2015-2021.

39. Sedaghat D, Zakir RM, Choe J, Klapholz M, Saric M. Cardiac amyloidosis in a patient with multiple myeloma: a case report and review of literature. J Clin Ultrasound 2009;37:179-184.

40. Abraham RS, Katzmann JA, Clark RJ, Bradwell AR, Kyle RA, Gertz MA. Quantitative analysis of serum free light chains. A new marker for the diagnostic evaluation of primary systemic amyloidosis. Am J Clin Pathol 2003;119:274-278.

41. Vrana JA, Gamez JD, Madden BJ, Theis JD, Bergen III R, Dogan A. Classification of amyloidosis by laser dissection and mass spectroscopy-based proteomic analysis in clinical biopsy specimens. Blood 2009; 114(24): 4957-4959.

42. Arbustini E, Merlini G, Gavazzi A, Grasso M, Diegoli M, Fasani R, Bellotti V, Marinone G, Morbini P, Dal Bello B, et al. Cardiac immunocyte-derived (AL) amyloidosis: an endomyocardial biopsy study in 11 patients. Am Heart J 1995 Sep;130:528-536.

43. Strege RJ, Saeger W, Linke RP. Diagnosis and immunohistochemical classification of systemic amyloidoses. Report of 43 cases in an unselected autopsy series. Virchows Arch 1998;433:19-27.

44. Brenner DA, Jain M, Pimentel DR, Wang B, Connors LH, Skinner M, Apstein CS, Liao R. Human amyloidogenic light chains directly impair cardiomyocyte function through an increase in cellular oxidant stress. Circ Res 2004; 94:1008-1010.

45. Liao R, Jain M, Teller P, Connors LH, Ngoy S, Skinner M, Falk $\mathrm{RH}$, Apstein CS. Infusion of light chains from patients with cardiac amyloidosis causes diastolic dysfunction in isolated mouse hearts. Circulation 2001;104:1594-1597.

46. Dispenzieri A, Kyle RA, Gertz MA, Therneau TM, Miller WL, Chandrasekaran K, McConnell JP, Burritt MF, Jaffe AS. Survival in patients with primary systemic amyloidosis and raised serum cardiac troponins. Lancet 2003;361:1787-1789.

47. Gertz MA, Falk RH, Skinner M, Cohen AS, Kyle RA. Worsening of congestive heart failure in amyloid heart disease treated by calcium channel-blocking agents. Am J Cardiol 1985;55:1645.

48. Khan MF, Falk RH. Amyloidosis. Postgrad Med J 2001; 77:686-693. 
49. Rubinow A, Skinner M, Cohen AS. Digoxin sensitivity in amyloid cardiomyopathy. Circulation 1981;63:1285-1288.

50. Dubrey S, Pollak A, Skinner M, Falk RH. Atrial thrombi occurring during sinus rhythm in cardiac amyloidosis: evidence for atrial electromechanical dissociation. Br Heart J 1995;74:541-544.

51. Santarone M, Corrado G, Tagliagambe LM, Manzillo GF, Tadeo G, Spata M, Longhi M. Atrial thrombosis in cardiac amyloidosis: diagnostic contribution of transesophageal echocardiography. J Am Soc Echocardiogr 1999;12:533-536.

52. Cohen AS, Rubinow A, Anderson JJ, Skinner M, Mason JH, Libbey C, Kayne H. Survival of patients with primary (AL) amyloidosis. Colchicine-treated cases from 1976 to 1983 compared with cases seen in previous years (1961 to 1973). Am J Med 1987;82:1182-1190.

53. Comenzo RL, Vosburgh E, Falk RH, Sanchorawala V, Reisinger J, Dubrey S, Dember LM, Berk JL, Akpek G, LaValley M, O'hara C, Arkin CF, Wright DG, Skinner M. Dose-intensive melphalan with blood stem-cell support for the treatment of AL (amyloid light-chain) amyloidosis: survival and responses in 25 patients. Blood 1998;91:3662-3670.

54. Kholova I, Kautzner J. Current treatment in cardiac amyloidosis. Curr Treat Options Cardiovasc Med 2006; 8:468-473.

55. Hrncic R, Wall J, Wolfenbarger DA, Murphy CL, Schell M, Weiss DT, Solomon A. Antibody-mediated resolution of light chain-associated amyloid deposits. Am J Pathol 2000; 157:1239-1246.

56. Grogan M, Gertz MA, Kyle RA, Tajik AJ. Five or more years of survival in patients with primary systemic amyloidosis and biopsy-proven cardiac involvement. Am J Cardiol 2000;85:664-665.

57. Hassan W, Al-Sergani H, Mourad W, Tabbaa R. Amyloid heart disease. New frontiers and insights in pathophysiology, diagnosis, and management. Tex Heart Inst J 2005; $32: 178-184$

58. Tanaka F, Migita K, Honda S, Fukuda T, Mine M, Nakamura T, Yamasaki S, Ida H, Kawakami A, Origuchi T, Eguchi K. Clinical outcome and survival of secondary (AA) amyloidosis. Clin Exp Rheumatol 2003;21:343-346.

\section{Author Affiliations}

Satya S. Bhupathi, MD, MPH*;

Sreelatha Chalasani, $M D, M P H^{*}$;

Roxann Rokey, MD, FACC, FASE'

*Department of General Internal Medicine,

Marshfield Clinic, Marshfield, Wisconsin USA

tDepartment of Cardiology,

Marshfield Clinic, Marshfield, Wisconsin USA 